\title{
Korean Economic Condition Indicator Using a Neural Network Trained on the 1997 Crisis
}

\author{
Tae Yoon Kim ${ }^{1}$, Changha Hwang ${ }^{2}$ and Jongkyu Lee ${ }^{3}$ \\ ${ }^{1}$ Keimyung University, ${ }^{2}$ Catholic University of Daegu and \\ ${ }^{3}$ The Bank of Korea
}

\begin{abstract}
The main aim of this article is to develop an efficient indicator for Korean economic conditions based on its disastrous 1997 economic crisis experience. For this an artificial neural network, a well known tool for pattern recognition, is employed. The dynamic movements of the 1997 stock price index are divided into three patterns or intervals according to a "volatility" level and then presented to the neural network as a training set. It turns out that the crisis trained neural network has a surprisingly high degree of accuracy in judging the given economic condition, which strongly suggests that the post crisis Korean economy has been profoundly influenced by the 1997 crisis. This result might also be useful to other countries trying to build an early crisis warning indicator.
\end{abstract}

Key words: Economic condition indicator, economic crisis, neural network, volatility.

\section{Introduction}

The 1997 Korean economy crisis brought massive bankruptcies of financial and industrial systems over a very short period of time, which was a quite new experience for Korea at that time which had become accustomed to a steady economic growth track. This unprecedented and peculiar crisis brought large scale change not only to the Korean economy but also to her entire society, and since then much attention has been focused on study of the economic crisis from a theoretical prospect. In particular much effort has been made to build an appropriate model that could detect a possible crisis in advance.

In this article we develop a neural network (NN) indicator trained on the 1997 stock price index whose main function is to judge the given economic condition. Interestingly enough the trained NN performs quite well when it is applied to periods other than 1997. In other words such a crisis experience seems to dominate the stimulus-response mechanism of the Korean economy after 1997. Another useful aspect of our result is related to economic crisis forecasting. Most of the 
statistical methodologies for crisis forecasting ${ }^{1}$ require a large amount of data related to crisis which are usually difficult to obtain in reality because such a disastrous economic crisis occurs rarely or over a very short period of time. The indicator developed in this article could help resolve this issue by providing more crisis related data which come from crisis classification results of the trained NN.

In this article the Korean stock price index (KOSPI) is used to judge whether the economy reaches the crisis level. Therefore the basic presumption of this article is that KOSPI reflects the given economic condition fairly well. This is a valid assumption because it was observed before the 1997 crisis that the Korean stock market was sufficiently mature that its participants were able to carry out their deals based on their own prospects for the Korean economy (Lee (1993)). In the meantime it may be also meaningful to analyze the behavior of the stock market in responding to potentially possible crisis. Of course there are other economic variables other than KOSPI such as the exchange rate and interest rate. Kim and Moon (1998), however, noticed that among possible economic variables KOSPI showed the highest variability, which implies KOSPI is more sensitive to changes in economic conditions.

The neural network (NN) used in this article is the back-propagation (BP) algorithm (Bishop, 1995), a quite popular tool for pattern recognition, and hence the process of building economic indicators is transformed into a pattern recognition problem. The 1997 KOSPI data and the KOSPI data other than 1997 are used as a training set and a test set respectively. Surprisingly the crisis trained NN performs with quality accuracy in judging the given economic condition. In particular it is quite interesting that another crisis was diagnosed by the crisis trained NN when a huge Korean conglomerate Hyundai faced a severe cash liquidity problem in September of 2000, when one considering the controversy about another possible crisis at that time.

This paper is composed as follows. Section II contains a thorough analysis of the 1997 KOSPI as a pattern recognition problem which results in the trained NN. Section III provides applications of the crisis trained NN to KOSPI other than in 1997 and evaluation of its validity and accuracy. Section IV contains concluding remarks.

\section{Training a New Economic Indicator on the 1997 KOSPI}

\subsection{Analysis of $1997 \mathrm{KOSPI}$}

A rough look at the 1997 KOSPI ( $x_{t}$, Figure 1) reveals that it starts at around 650 and reaches up to 800 in June and then continues to fall rapidly

\footnotetext{
${ }^{1}$ Signal approach is employed by Kaminsky et al.(1997), regression method by Berg and Pattillo(1998), and severity of crisis indicators by Sachs et al.(1996) and Glick and Rose(1998).
} 
without any noticeable upturn until December in which Korea was officially put on the IMF (International Monetary Fund) financial rescue program. To check a structural change of the $1997 \mathrm{KOSPI}$, daily rise and fall rate $p_{t}=\left(x_{t}-x_{t-1}\right) / x_{t-1}$ is calculated. The main reason why $p_{t}$ is chosen here is that increased instability or volatility of the stock market due to the potentially possible crisis is expected to eventually lead to a sudden increase of frequency and amplitude of $p_{t}$. As expected such phenomenon is easy to notice in Figure 3. Indeed from September of 1997 the frequency and amplitude of $p_{t}$ starts to increase noticeably, which is quite similar to a sudden increase of frequency and amplitude of seismic waves as an earthquake comes close.

To investigate further into $p_{t}$, its 10 day moving average $\bar{p}_{t}=\frac{1}{10} \sum_{i=t-9}^{t} p_{i}$ and 10 day moving variance $s_{t}^{2}=\frac{1}{10} \sum_{i=t-9}^{t}\left(p_{i}-\bar{p}_{t}\right)^{2}$ (Figure 3 , another measure of volatility) are studied. A rather short period of 10 days was chosen here for the moving average to take into account the visibly clear nonstationarity from Figure 3. We note that there is not much difference between $\bar{p}_{t}$ and $p_{t}$, which implicitly suggests the choice of 10 days is appropriate. Figure 3 shows that $s_{t}^{2}$ starts to increase from September of 1997 when KOSPI is believed to be responding to the potentially possible crisis.

To find more specific dates of sudden volatility increase, moving variance ratio $r_{t}=s_{t}^{2} / s_{t-1}^{2}$ (Figure 4) is obtained because a sudden increase of moving variance $s_{t}^{2}$ could be a critical signal for the possible crisis. In fact there was an obvious signal at September 30 of 1997 whose $r_{t}$ exceeds 4, which is almost two months earlier than December 3, the official crisis date acknowledged by IMF.

Using the KOSPI analysis above, the year 1997 is divided into three intervals or patterns. Specifically i) January 3 through September 18 is termed as the stable period (SP), (ii) September 19 through October 21 with the signal September 30 as its center is termed as the unstable period (UP), (iii) October 22 through December 27. the crisis period (CP). SP is literally a stable period and UP is a period which is characterized by a sudden increase of volatility and rapid swings in market sentiment. In $\mathrm{CP}$ the stock market recognizes the occurrence of the economic crisis and adapts itself to the crisis. Since these three periods or patterns are structurally different from each other ${ }^{2}$, they are considered as a pattern set to be classified and thus serve as a basis of the economic indicator developed in this section. The terms stable, unstable and crisis periods are used as descriptive terminologies to describe the stock market responses to the given economic conditions. There is no clear cut consensus about the definition of an economic crisis but we establish our definition in terms of volatility in this paper.

The unstable period above, usually occurring just prior to a crisis, could be interpreted as a period through which the economy makes a transition from a

\footnotetext{
${ }^{2}$ EDA and training result of Section III.2 may be referred to in this regard.
} 


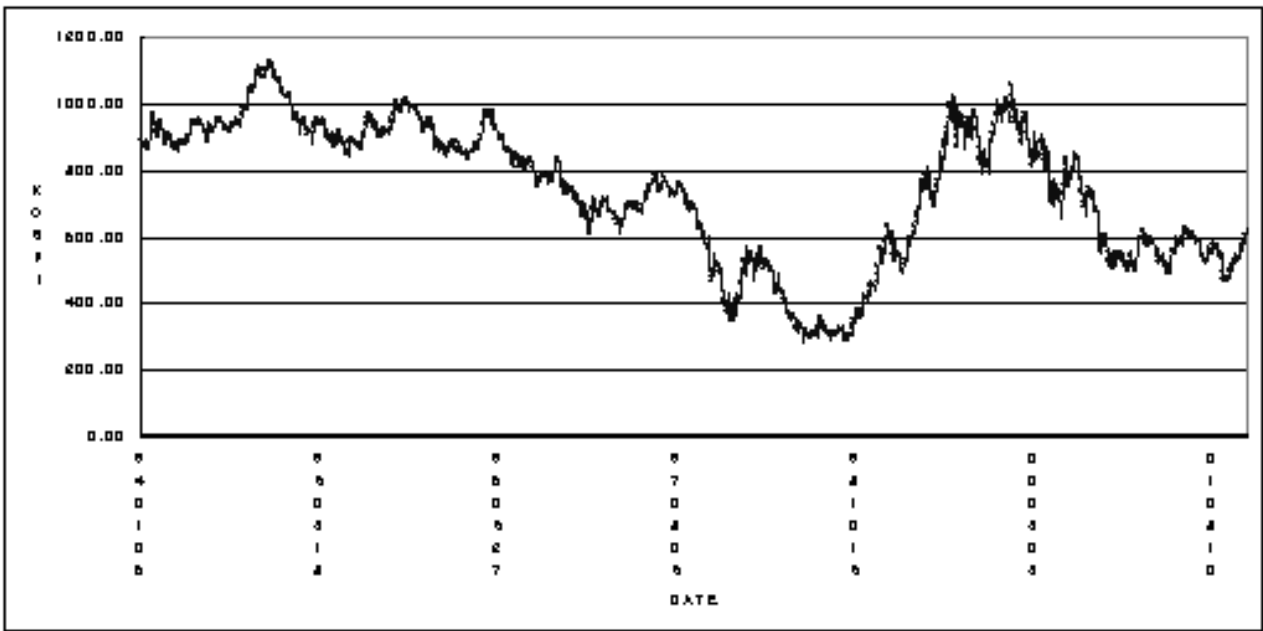

Figure 1: KOSPI from January 1994 to November 2001

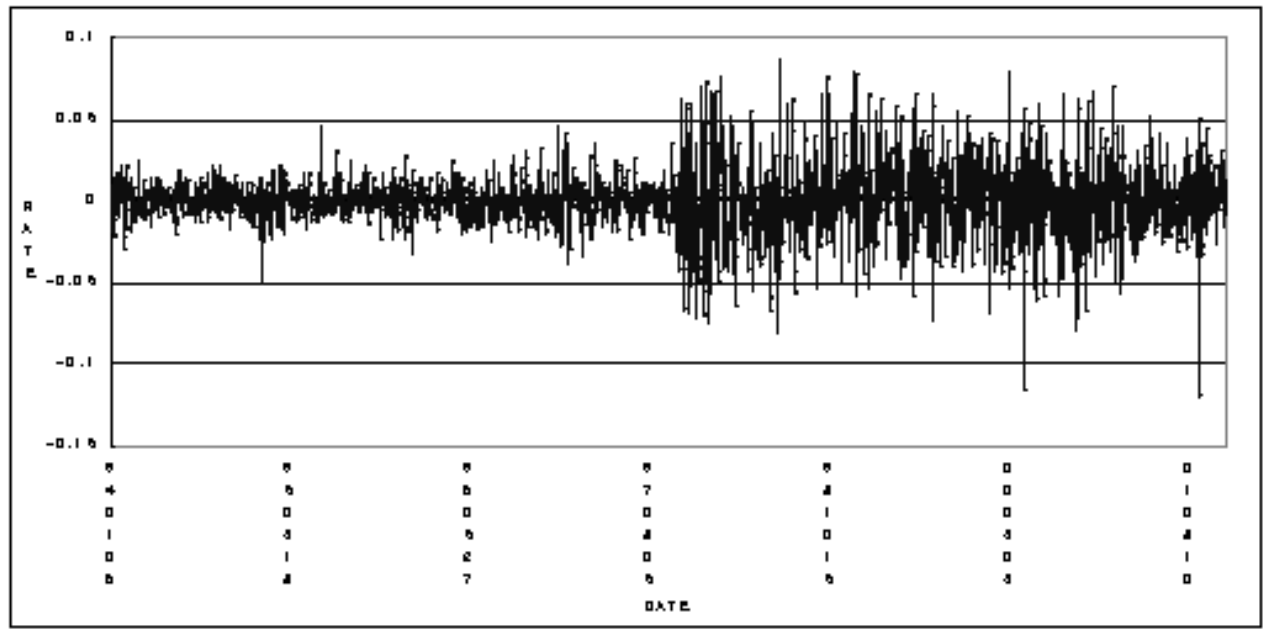

Figure 2: Daily rise and fall rate, $p_{t}=\left(x_{t}-t_{t-1}\right) / x_{t-1}$.

stable condition to crisis. Often it is called a gray zone where the economy loses self-correcting mechanisms, see Lee (2000) for more details. There is no consensus about how long it lasts but it is usually expected to be very short because of an abrupt reversal of market sentiment. In the mean time a gray zone may proceed to either a stable condition or a crisis. In other words even though the economy reaches to gray zone, it may get back to a stable one through an appropriate recovery or reform measures. One of the main contributions of our result is that the crisis trained indicator is able to provide an efficient decision about whether the economy has entered the gray zone. 


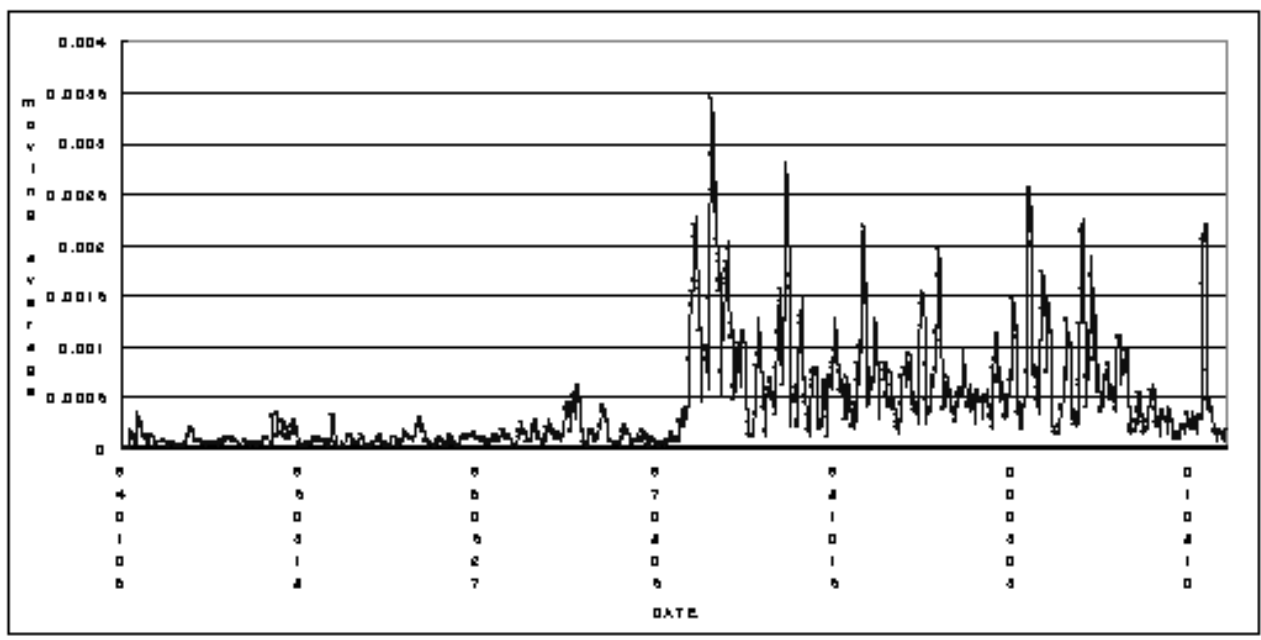

Figure 3: Moving variance of rise and fall rate $s_{t}^{2}=\sum_{i=t-9}^{t}\left(p_{i}-\overline{p_{t}}\right)^{2} / 10$.

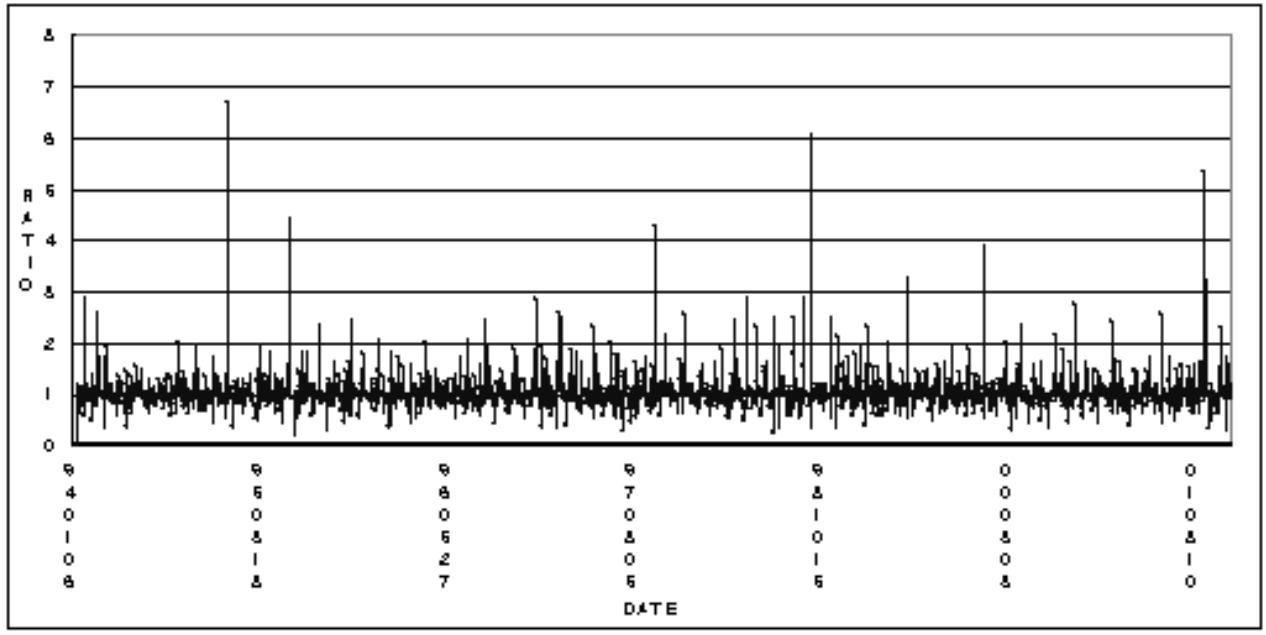

Figure 4: Ratio of moving variance, $\gamma_{t}=s_{t}^{2} / s_{t-1}^{2}$.

\subsection{The crisis trained neural network}

Training on the 1997 crisis KOSPI is done to the well known back propagation neural network which consists of an input layer of 5 nodes, hidden layer of 7 nodes and output layer of three nodes (Figure 5). Here 5 input variables $\left(x_{t}, p_{t}, \bar{p}_{t}, s_{t}^{2}\right.$, $r_{t}$ ) are assigned to each input node. Input variable selection is done mainly via visual time plot analysis (see Section 2.1) but the validity of 5 input variables 


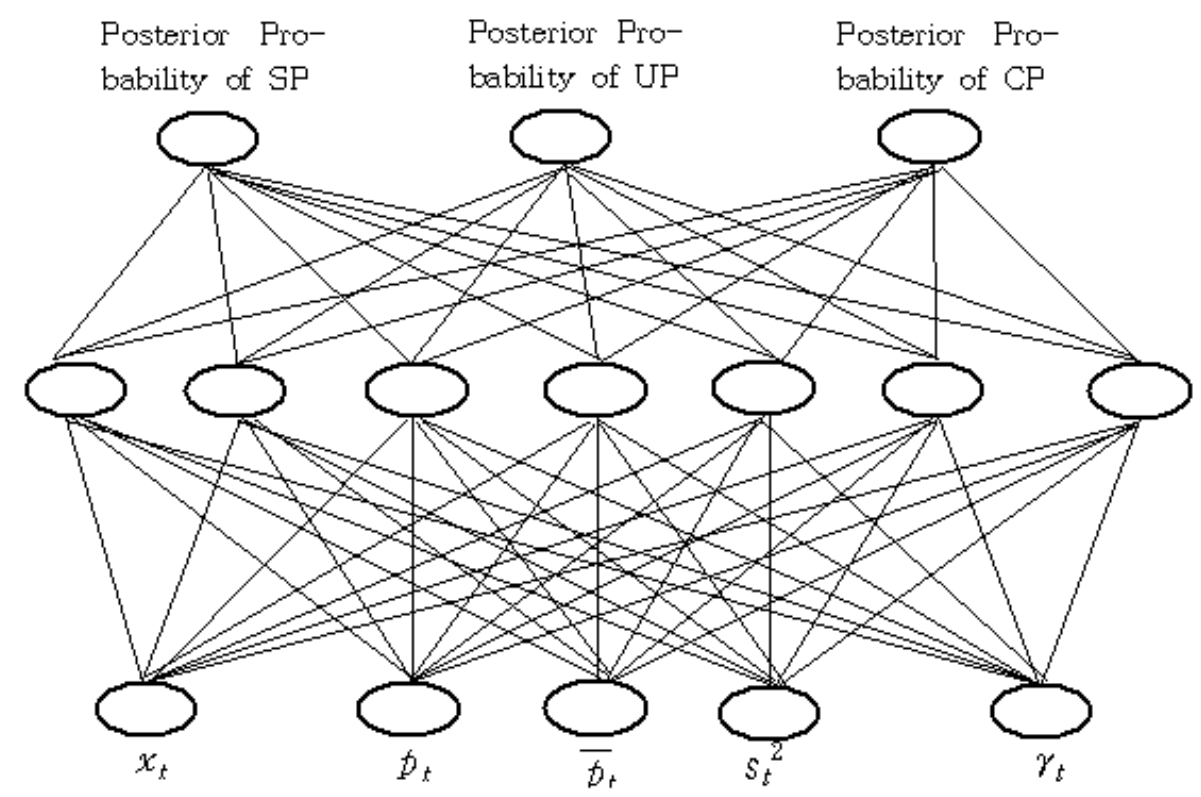

Figure 5: Neuro network employed.

is checked through EDA analysis such as principal component or cluster analysis (see Kim et al. (2004) for detail.). The results of EDA analysis can be summarized as follows. $x_{t}$ and $s_{t}^{2}$ pick up distinction among three patterns efficiently while $p_{t}$ is particularly successful at distinguishing between SP and the remaining periods. Of course $r_{t}$ is good at distinguishing between UP and the remaining ones. With these EDA and graphical analysis at hand, it seems desirable to select $x_{t}, s_{t}^{2}, p_{t}$ and $r_{t}$ as input variables. Finally $\bar{p}_{t}$ is added since it would make more sense to have the 10 day moving average (a past history) instead of having just current daily data as input variables. Logistic classification and decision tree procedures were employed to check the validity of the input variables, which led to an almost identical result to EDA. Posterior probabilities of SP, UP, and CP are assigned to each node of output layer and the one with the highest posterior probability is to be picked as the final output of $\mathrm{NN}$, i.e., the period (or pattern) to which the given economic condition belongs.

Interestingly the error rate of the $\mathrm{NN}$ with 5 input variables on the training sample was zero percent ${ }^{3}$ after it was trained on the 1997 KOSPI (refer $T_{2}$ of

\footnotetext{
${ }^{3} \mathrm{SAS}$ E-miner $\mathrm{NN}$ is implemented with initial weights given by random number generator from $\mathrm{U}(-1 / 2,1 / 2)$. It is possible to have a slightly different error rate than 0 percent under a different training environment.
} 
Figure 6). Support vector machine $(\mathrm{SVM})^{4}$ was implemented to check an overfitting of $\mathrm{NN}$ (0 percent error rate of $\mathrm{NN}$ often indicates a possible overfitting) and it also produced a 0 percent error rate.

\section{Application of the Crisis Trained NN}

\subsection{Analysis of test data set}

Test data set consists of data from January 4 of 1994 to November 22 of 2001 other than 1997. A rough look at the KOSPI plot over the period (Figure 1) reveals the followings. It has reached its peak at 1150 in November of 1994 and then continue to fall on average until December of 1997. There was a small rally in early 1998 but the downward trend prevailed until September of 1998 when a real rally seems to have started and continued until early 2000. After that, the downturn trend comes back in again.

The rise and fall rate $p_{t}$ of the above mentioned period (Figure 2) discloses that frequency and amplitude of $p_{t}$ is increased sharply as a crisis approaches, which confirms the fact that a sudden increase in volatility in a stock market mainly due to unrest may be a good indicator of a possible crisis. Also one may note that its frequency returns to its previous normal level after a lapse of some time but its amplitude continues to stay at the crisis level. That the amplitude does not return to its previous level is probably due to the fact that to overcome the crisis takes a long period of time and the Korean economy went through structural change via its economic reform program ${ }^{5}$ after the crisis. The ratio $r_{t}$ (Figure 4) has several flags whose values exceed 4, i.e., 1995 January 4, 1995 May 29, 1997 September 30, 1998 September 24, 2000 January 5 and 2001 September 12. These flags could be explained in the context of economic conditions around those dates. ${ }^{6}$ Indeed January 4, 1995 seems to be the result of government announcements of self-regulating and advancement measures for the stock market, May 29, 1995 the result of government announcements of stock market stabilization measures, and September 30, 1997 the signal of the coming crisis. September 24, 1998 seems to be a reflection of a possible US interest rate

\footnotetext{
${ }^{4} \mathrm{SVM}$ is devised mainly to resolve shortcomings of NN like local minima, slow convergence rate and overfitting problems, See Burges (1998).

${ }^{5}$ Daily upper and lower limits of individual stock price movements were eased from $12 \%$ to 15 $\%$ after the crisis which was a part of economic reform program led by the Korean government. Further research seems to be necessary about the impact of such measures on the crisis trained indicator. Considering the accuracy and stability of the indicator around those price limit adjustment dates, however, the indicator does not appear to be influenced severely by price limit adjustment measures. Those price limit adjustment dates are 95.1.14, 96.11.25, 98.2.3 and 98.7.12.

${ }^{6}$ The Bank of Korea (2000) may be useful in this connection.
} 
cut (alluded to by Mr. Greenspan) and improvements of domestic and the US economic conditions and January 5, 2000 seems to be due to apprehension of a possible US interest rate raise. The September 12, 2001 is the direct result of the terrorist attacks against the World Trade Center in New York and the Pentagon. These flag analysis suggests a constitutional change of the Korean stock market, i.e., after the crisis it reacts more sensitively to changes in economic conditions than government policy announcements.

\subsection{Application and accuracy of the crisis trained indicator}

In this section we apply the crisis trained economic condition indicator to the period other than 1997 and evaluate their accuracy. The results of classifications of the indicator are given in Figure 6. where 1, 2 and 3 denote SP (stable period), $\mathrm{UP}$ (unstable period), and $\mathrm{CP}$ (crisis period), respectively.

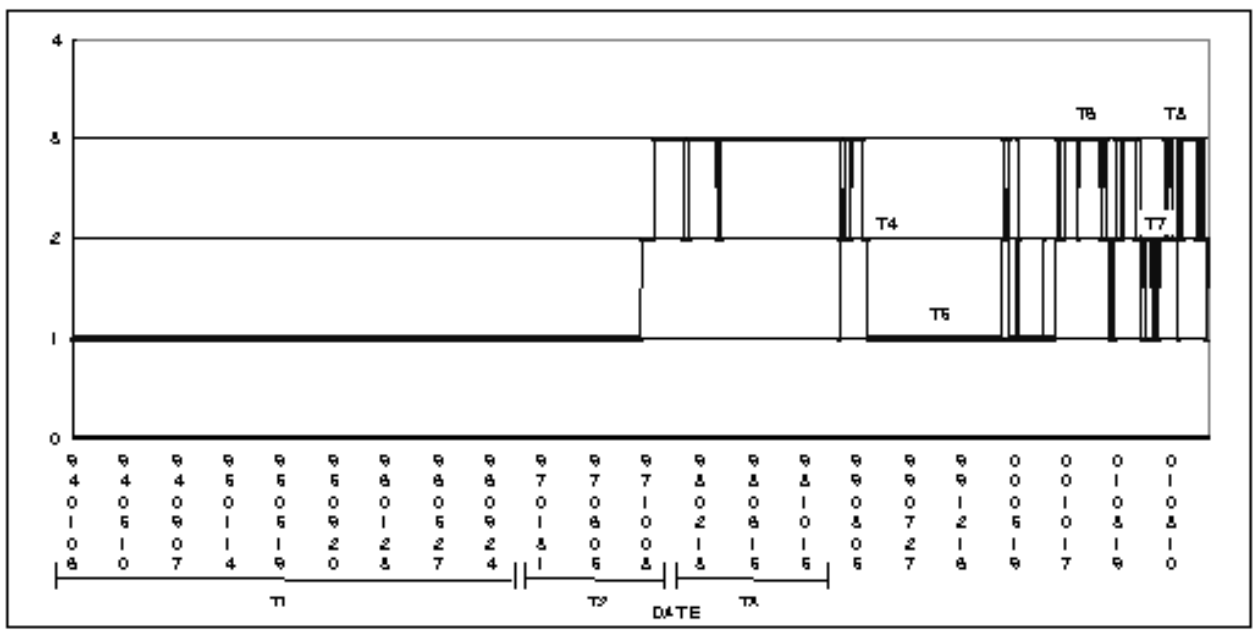

Figure 6: Classification resuts.

As discussed earlier, after training is done, $T_{2}$ (Jan. 1997 - Dec. 1997) is classified correctly with a very high degree of accuracy ${ }^{7}$. First note that $T_{1}$ (Jan. 1994 -Dec. 1996) is correctly classified as expected. T3 (Jan. 1998- Mar. 1999) is classified as CP and $T_{5}$ (Apr. 1999-Sep. 2000) enjoys SP after $T_{4}$ (Mar. 99-Apr. 99) stays on UP briefly. $T_{6}$ (Sep. 2000-Apr. 2001) is classified as CP again and then $T_{7}$ (May 2001- Sep. 2001) and $T_{8}$ (Sep. 2001- Nov. 2001) are classified as UP and CP respectively. Since the classification is done by finding the period

\footnotetext{
${ }^{7}$ Classification results of $T_{2}$ are given by training the neural network.
} 
or pattern which maximizes the posterior probabilities, more often than not it is observed that the classified period move fast between the patterns (for example, the latter part of $T_{3}$, a few points of $T_{6}, T_{7}$ and $T_{8}$ ). This is simply because those points are difficult to classify with the trained NN but they may be ignored in finding the overall trend of the Korean economy over years.

These classification results appear to coincide with the real economic trend that Korea followed. Indeed on $T_{3}$ right after the 1997 crisis, the Korean economy suffered many difficulties but overcame them through a strong economic reform program and the steady and strong US economic expansion. As a result it returned to the stable condition on $T_{5}$. One noteworthy thing here is that it went through a very short UP (unstable period) on $T_{4}$ during transition from $\mathrm{CP}\left(T_{3}\right.$ ) to $\mathrm{SP}\left(T_{5}\right)$, which shows that a sudden increase of volatility may occur when a favorable transition from CP to SP is expected. Since September of 2000, the Korean economy has experienced difficulties due to a severe liquidity problem of a huge Korean conglomerate, Hyundai, and the worsening of US economic conditions. The crisis trained indicator classifies those periods $\left(T_{6}\right.$ and $\left.T_{7}\right)$ as $\mathrm{CP}$ and UP. This suggests that the economy may become unstable when reform measures to prevent a crisis are not taken properly. (There was a heated debate about a backfiring of economic reform in March of 2001.) One may note a rather long unstable period $\left(T_{7}\right)$ after $T_{6}$. Finally another crisis came on $T_{8}$ which was the immediate result of the terrorists attacks against the US on Sep. 11th, 2001.

It is interesting to see that classification as CP of 2000.9 comes right after SP classification, i.e., there was no in-between UP classification in the transition from SP to CP. This contrasts with the 1997 crisis having a rather long UP in-between SP and CP. The main difference is as follows. In 1997 there was a strong government public relation campaign which firmly denied any possibility of crisis in the near future and eventually resulted in a rather long UP before the crisis, while in 2000 there was no such strong public relation campaign by the government and the Korean economy, having experienced the 1997 crisis, reacted swiftly to the coming crisis. Note that the 2001 crisis $\left(T_{8}\right)$ is one from a purely external incident.

In summary, applications of the crisis trained indicator to the test data show a very accurate classification results overall in the sense that they almost coincide with the real economic trend that Korea followed.

\section{Concluding Remarks}

In this paper, an economic condition indicator is trained on the Korean stock price index (KOSPI) over the 1997 crisis which is divided into three patterns, i.e., a stable, unstable, and crisis period. Applications of the indicator to test data display surprisingly high accuracy. The main reason is that the 1997 crisis 
experience seems to have set a benchmark for all sectors of Korean economy including the government.

\section{References}

Bishop, C. M. (1995). Neural Networks for Pattern Recognition. Clarendon Press. Oxford.

Berg, A. and Pattillo, C. (1999). Are currency crises predictable? A test. IMF working papers 46.

Burges, C. (1998). A tutorial on support vector machines for pattern recognition. In Data Mining and Knowledge Discovery 2. Kluwer Academic Press.

Glick R. and Rose, A. K. (1998). Contagion and trade: Why are currency crises regional? NBER Working Paper 6806.

Kaminsky, G., Lizondo, S. and Reinhart, C. M. (1997). Leading indicators of currency crisis. IMF working paper $97 / 79$.

Kim, M. and Moon, S. (1998). Mutual association between exchange rate interest rate and KOSPI. Economy Analysis 4, 93-113. (in Korean).

Kim, T. Y., Oh, K. J., Sohn, I. and Hwang, C. (2004). Usefulness of artificial neural networks for early warning systems of economic crisis. Expert Systems with Applications 26, 583-590.

Lee J. (2000). Economic Crises: Their Causes and Processes. Finance and Economy Collection, Economic Studies Office, The Bank of Korea. (in Korean).

Lee, M. (1993). Korean stock market efficiency and KOSPI analysis. Finance and Economics 62, The Bank of Korea (in Korean).

Sachs, J., Tornell, A. and Velasco, A. (1996). Financial crises in emerging markets: The lessons from 1995. Brookings Papers on Economic Activity.

The Bank of Korea (2000). Financial and Economic Chronicles of Korea. (in Korean).

Received March 2, 2003; accepted July 25, 2003.

Tae Yoon Kim

Department of Statistics

Keimyung University

Taegu 704-701, Korea

Changha Hwang

Department of Statistical Information

Catholic University of Daegu

Taegu 713-702, Korea 
Jongkyu Lee

Economic Studies Office

The bank of Korea

Seoul 100-794, Korea 